\title{
Modificación en el esquema de vacunación antipertussis en Chile, vacunación en grupos especiales y estrategias de control. Comentario del Comité Consultivo de Inmunizaciones (CCI) de la Sociedad Chilena de Infectología
}

\author{
Marcela Potin, Jaime Cerda, Lily Contreras, Alma Muñoz, Erna Ripoll y Rodrigo Vergara \\ en representación del Comité Consultivo de Inmunizaciones de la Sociedad Chilena de Infectología)
}

Modification of pertussis vaccination schedule in Chile, immunization of special groups and control strategies. Commentary from the Consultive Committee of Immunizations of The Chilean Society of Infectious Diseases

In Chile, an increased number of notifications of cases of whooping cough was detected at the beginning of October 2010, and maintained through 2012. Accumulated cases during 2011 were 2,581 (15.0 per 100,000), which is greater than the number of cases registered during the period 2008-2010 (2,460 cases). On the other hand, the local sanitary authority introduced a modification of pertussis vaccination schedule (starting 2012), which consists in the replacement of the second booster of pertussis vaccine (DTwP, administered to 4-year-old children) as well as diphtheria-tetanus toxoid (dT, administered to second grade scholars) for an acellular pertussis vaccine with reduced antigenic content (dTpa), which will be administrated to first grade scholars. The Consultive Committee of Immunizations considers that the modification is adequate, since it extends the age of protection, reducing at least in theory the infection in older scholars and adolescents - who are significant sources of transmission of Bordetella pertussis to infants- using an adequate vaccine formulation (acellular pertussis vaccine). The available evidence regarding vaccination in special groups (adolescents and adults, health-care workers and pregnant women) and cocooning strategy are commented.

Key words: Bordetella pertussis, whooping cough, pertussis, vaccines.

Palabras clave: Bordetella pertussis, coqueluche, tos ferina, vacunas.

\begin{tabular}{|ll|}
\hline \multicolumn{1}{c|}{ Abreviaturas } \\
\hline DTwP: & $\begin{array}{l}\text { vacuna anti diftérica/anti tetánica/antipertussis de } \\
\text { células enteras }\end{array}$ \\
dTpa: & $\begin{array}{l}\text { anti diftérica/vacuna anti tetánica/antipertussis } \\
\text { acelular de contenido antigénico reducido }\end{array}$ \\
Vacuna & DTwP + hepatitis B + H. influenzae tipo b \\
pentavalente: & Comité Consultivo de Inmunizaciones \\
CCl: & \\
\hline
\end{tabular}

\section{Introducción}

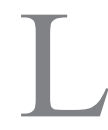

a coqueluche o tos ferina es una enfermedad causada por la bacteria Bordetella pertussis, la cual es transmitida desde sujetos infectados a susceptibles a través de gotitas. En su etapa catarral temprana la coqueluche es altamente transmisible, con una tasa de ataque secundaria de hasta $90 \%$ entre contactos no-inmunes al interior del hogar. La vacunación a gran escala realizada durante las décadas de 1950 y 1960 produjo una reducción mayor al $90 \%$ en la incidencia y mortalidad por esta causa en países industrializados. En el año 2008, según informes de la Organización Mundial de la Salud, cerca de $82 \%$ de los niños alrededor del mundo recibieron tres dosis de vacuna anti-pertussis, estimándose que la vacunación evitó aproximadamente 687.000 muertes $^{1}$.

Dos hechos ocurridos en Chile a fines de 2010 marcaron la agenda en el control de la infección por B. pertussis. El primero, de carácter epidemiológico, tiene relación con el aumento en el número de casos de coqueluche en nuestro país. Los casos registrados durante 2011 ascendieron a $2.581^{2}$, cifra superior a la sumatoria de casos registrados en el período $2008-2010$ (2.460 casos $)^{3}$. El segundo tiene relación con la consecuente modificación introducida por la autoridad sanitaria en el esquema de vacunación anti-pertussis, consistente en el reemplazo del segundo refuerzo de vacuna anti-pertussis (DTwP, administrada a los 4 años) y del toxoide diftérico-tetánico (dT, administrado en segundo básico) por una vacuna anti-pertussis acelular con contenido antigénico reducido (dTpa), a ser administrada en el primer año de enseñanza básica. El presente documento tiene como objetivo describir la situación
División de Pediatría. Facultad de Medicina, Pontificia Universidad Católica de Chile (MP). Departamento de Salud Pública. Facultad de Medicina, Pontificia Universidad Católica de Chile (JC) Servicio de Pediatría, Hospital de Carabineros. Universidad Mayor. Santiago-Chile (LC, AM). Departamento de Enfermedades Respiratorias y Alergias, CENTRO MED. Viña del Mar-Chile (ER). Departamento de Preclínicas y Pediatría. Escuela de Medicina, Universidad de Valparaíso (RV).

Declaración de conflictos de interés: Marcela Potin ha recibido honorarios por charlas de vaccinología general para GSK y Sanofi Pasteur, y ha recibido honorarios en comité de expertos para Pfizer. Jaime Cerda y Erna Ripoll reportan no presentar conflictos de interés. Lily Contreras ha recibido apoyo de GSK, Sanofi Pasteur, Merck y Pfizer para asistencia a congresos científicos.

Alma Muñoz ha recibido financiamiento por concepto de estudios clínicos de GSK, Novartis, Medimmune, Pfizer y Sanofi Pasteur; ha asistido a congresos y reuniones científicas con el auspicio de GSK, Sanofi Pasteur, Novartis, Berna Pfizer y ha recibido honorarios de GSK en consultoría de expertos. Rodrigo Vergara ha recibido financiamiento de GSK y Novartis para estudios clínicos de vacunas distintas a vacunas contra pertussis, ha asistido a congresos y reuniones científicas con auspicio de GSK y Novartis, ha recibido honorarios de GSK por consultoría de expertos $y$ de Merck por charla de vacunas distintas a vacunas antipertussis.

Correspondencia a: Marcela Potin Santelices mpotin@med.puc.cl 
epidemiológica de la infección por $B$. pertussis en Chile, comentar la modificación introducida por la autoridad sanitaria a partir de 2012 en el esquema de vacunación anti-pertussis y revisar la evidencia actualizada respecto a la vacunación de tres grupos especiales (adolescentes y adultos, funcionarios de la salud y mujeres embarazadas), así como la estrategia de vacunación en capullo. Como el presente artículo se refiere solamente a estrategias para el control de la coqueluche basadas en el uso de vacunas, remitimos al lector interesado en otros importantes aspectos relacionados al control de esta enfermedad a la circular emitida por el Departamento de Epidemiología del Ministerio de Salud, con fecha 5 de julio de 2011 (Circular B51/N²7), titulada Vigilancia Epidemiológica y Medidas de Control de Coqueluche. Este documento reemplaza la circular B51/No 4 del 9 de febrero del 2007 y resume aspectos clínicos, epidemiológicos, medidas de control, tratamiento, enfrentamiento de brotes, aspectos de laboratorios y acciones preventivas vigentes en Chile $\mathrm{e}^{4}$.

\section{Situación epidemiológica de la infección por Bordetella pertussis en Chile}

En nuestro país, la infección por $B$. pertussis es de carácter endémico. Luego de registrarse un alto número de casos en el año 2000 (3.281 casos; 21,3/100.000 hab.), éstos descendieron y se estabilizaron durante el período 2002-2010, observándose una mediana de 1.056 casos anuales y una mediana de incidencia de 6,6/100.000 hab. ${ }^{3,5}$ (Figura 1). Sin embargo, a comienzos del mes de octubre de 2010 se detectó un aumento en la notificación de casos de coqueluche, dinámica que se ha mantenido a la fecha. El número de casos acumulados durante 2011 ascendió a

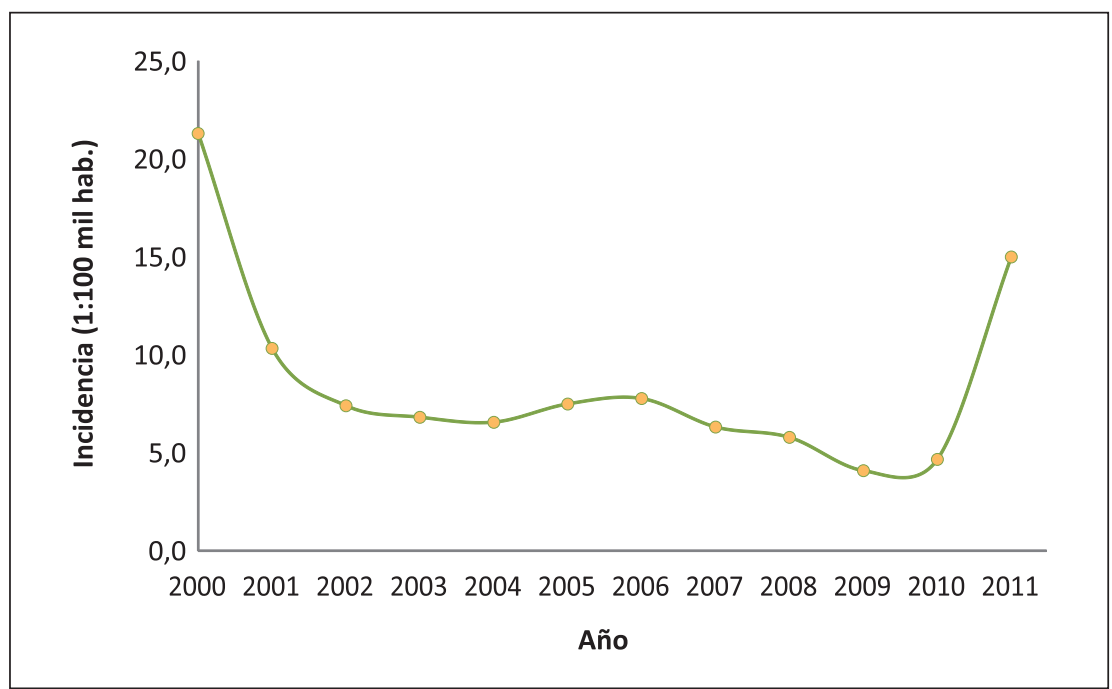

Figura 1. Tasa de incidencia de coqueluche (Chile, 2010-2011). Fuente: Elaboración propia, a partir de datos reportados por el Departamento de Estadísticas e Información en Salud ${ }^{3}$ e Instituto Nacional de Estadísticas 5 .
$2.581(15,0 / 100.000 \text { hab. })^{2}$, cifra superior a la sumatoria de casos registrados durante 2010 (798 casos), 2009 (692 casos) y 2008 (970 casos $)^{3}$. En un análisis por regiones, las mayores tasas de incidencia acumulada se registraron en la Región del Bío-Bío (40,1/100.000 hab.) y en la Región Metropolitana (15,6/100.000 hab.). Otras regiones tales como Valparaíso, Del Libertador B. O’Higgins, Maule, Los Ríos y Los Lagos, también presentaron un aumento destacable en sus notificaciones, por sobre la mediana del quinquenio anterior ${ }^{2}$. Globalmente, $1.295(50,2 \%)$ de los casos de coqueluche notificados en Chile durante 2011 correspondieron a lactantes bajo 1 año de edad; de ellos, $79,1 \%$ tenían menos de 6 meses de edad (i.e. lactantes que no alcanzaron a completar su serie primaria de vacunación anti-pertussis) ${ }^{2}$. El número de fallecidos por coqueluche durante 2011 fue de 16 personas, todos lactantes bajo 6 meses de edad ${ }^{2}$. Los casos acumulados al 14 de abril de 2012 (hasta el término de la semana epidemiológica 15) ascienden a 838 , cifra superior a igual período en 2011 (419 casos). La Región del Bío-Bío da cuenta de 43\% de los casos (365), manteniendo la mayor incidencia a lo largo del país. Se han reportado 5 fallecidos, todos bajo 6 meses de edad 6 .

\section{Vacunación anti-pertussis en Chile y modificación introducida a partir de 2012}

El esquema de vacunación anti-pertussis utilizado en nuestro país incluye una serie primaria de tres dosis $(2$, 4 y 6 meses; vacuna pentavalente), un primer refuerzo (18 meses; vacuna pentavalente) y un segundo refuerzo (4 años; vacuna DTwP). Por su parte, en el segundo año de enseñanza básica se administra un refuerzo de toxoide diftérico-tetánico (dT). Todas las vacunas anti-pertussis utilizadas corresponden a vacunas de células enteras. A partir de 2012, según se detalla en el Ordinario B27 N4006 emitido por la Subsecretaría de Salud Pública y la Subsecretaría de Redes Asistenciales ${ }^{7}$, se reemplaza el segundo refuerzo de vacuna anti-pertussis y el refuerzo de toxoide dT por la vacuna dTpa, a ser administrada en primer año de enseñanza básica. La autoridad sanitaria señala que la modificación del esquema entrará en vigencia a contar de la emisión del decreto correspondiente.

\section{Comentario del CCI a la modificación introducida a partir de 2012 en el esquema de vacunación anti-pertussis}

Para poder calificar la idoneidad de la modificación en el esquema de vacunación anti-pertussis, dos antecedentes deben ser tomados en consideración.

- En Chile, la cobertura de vacunación anti-pertussis (vacuna pentavalente, administrada a los 6 meses de edad) 
alcanzó a 94,9\% (2008) y 93,5\% (2010) ${ }^{8}$. A los dos años de edad (i.e. con posterioridad a la administración del primer refuerzo) el porcentaje es de 88,2\% (2009), situándose por debajo del promedio de los países de la $\operatorname{OCDE}(95,3 \%)^{9}$. Poco se sabe respecto a la duración de la protección que confiere la vacunación anti-pertussis en países en vías de desarrollo; sin embargo, estudios realizados en países industrializados han demostrado que la inmunidad declina luego de 4 a 12 años ${ }^{10}$.

- En numerosos países el reporte de casos de coqueluche en niños mayores, adolescentes y adultos ha experimentado un alza progresiva, constituyendo importantes fuentes de contagio de $B$. pertussis a lactantes no vacunados. Por desgracia, con frecuencia la coqueluche no es diagnosticada en niños mayores, adolescentes y adultos debido a su presentación atípica ${ }^{1}$, situación que también ha sido documentada en nuestro medio ${ }^{11-14}$. La evidencia epidemiológica apoya el supuesto de que la pérdida de inmunidad que ocurre con la edad, explica, al menos parcialmente, el aumento de incidencia de coqueluche en escolares, adolescentes y adultos ${ }^{1}$.

Hasta 2011, el esquema de vacunación anti-pertussis incluía un segundo y último refuerzo a los 4 años. El hecho de retrasar la administración del segundo refuerzo hacia el primer año de enseñanza básica, permite extender el tiempo de protección, reduciendo, al menos en teoría, la infección en escolares mayores y adolescentes, quienes son importantes fuente de contagio para los lactantes. Por su parte, la decisión de administrar la vacuna dTpa parece ser adecuada; al respecto, la OMS señala que (1) en personas sobre 6 años de edad, solamente deben utilizarse vacunas acelulares, y que (2) realizar cambios de formulación entre vacunas anti-pertussis de células enteras y acelulares es poco probable que interfiera en la seguridad e inmunogenicidad de estas vacunas ${ }^{1}$.

Por último, es importante destacar una ventaja asociada a la modificación del esquema de vacunación anti-pertussis. Si bien no existe evidencia alguna de eventos adversos en lactantes o niños por exposición al timerosal contenido en vacunas rutinarias ${ }^{15}$, basándose en un principio precautorio, el Ministerio de Salud progresivamente ha adoptado formulaciones de vacunas con menores cantidades de timerosal; de hecho, desde mediados del año 2010, la vacuna pentavalente administrada en nuestro país actualmente es una formulación con reducción de timerosal y contiene entre 1 y $2 \mu \mathrm{g}$ por dosis de vacuna, los que son necesarios para mantener su esterilidad en las presentaciones multidosis. Las vacunas DTwP y dT, hasta ahora administradas a los 4 y 7 años respectivamente, contienen $25 \mu \mathrm{g}$ de timerosal/dosis de vacuna. Estas dos vacunas serán reemplazadas por la vacuna dTpa, libre de timerosal.

\section{Evidencias sobre el beneficio de vacunación anti-pertussis en grupos especiales}

\section{Vacuna anti-pertussis en adolescentes y adultos}

La OMS considera que existe evidencia insuficiente para apoyar la incorporación de dosis de refuerzo en adolescentes y adultos para reducir la coqueluche grave del lactante. Sin embargo, reconoce que la vacunación puede prevenir la enfermedad en adolescentes y adultos. Por su parte, el Comité Asesor en Prácticas de Inmunización (Advisory Committee on Immunization Practices- ACIP) recomienda la administración de una dosis única de dTpa en adolescentes entre 11-18 años que hayan completado su serie de vacunación infantil, y en adultos entre los 19-64 años. Asimismo, recomienda la administración de una dosis única de dTpa a adultos de 65 o más años que tengan o vayan a tener contacto cercano con un lactante bajo 12 meses de edad, y señala que a otros adultos a partir de 65 años de edad, se les podría administrar una dosis única de $\mathrm{dTpa}^{16}$. En vista que ambos objetivos son válidos (reducir la coqueluche grave en lactantes y reducir los casos de coqueluche en adolescentes y adultos), como CCI consideramos que una modificación favorable de nuestro Plan Nacional de Inmunizaciones sería la incorporación progresiva de vacunas anti-pertussis (dTpa) en adolescentes y adultos, siendo necesario definir los momentos en que habrían de administrarse estas vacunas, en el contexto de nuestro Plan de Inmunización.

\section{Vacunación de funcionarios de la salud}

En opinión de la OMS, la vacunación de funcionarios de la salud para prevenir la transmisión nosocomial a lactantes y personas inmunocomprometidas puede ser costo-efectiva si se alcanzan altas coberturas. La vacuna estaría recomendada especialmente en funcionarios que se desempeñan en maternidades y unidades pediátricas ${ }^{1}$. Al respecto, la experiencia nacional reportada es escasa, pero alentadora. Durante el mes de abril de 2011 la Red de Salud de la P. Universidad Católica de Chile ofreció en forma gratuita vacunación anti-pertussis (dTpa) a 455 funcionarios de salud que trabajan en contacto con pacientes pediátricos en áreas hospitalarias, ambulatorias y de laboratorios. 355 sujetos $(83,7 \%)$ aceptaron vacunarse, siendo la aceptabilidad similar según estamento (médicos $81 \%$, enfermeras $73 \%$, técnicos paramédicos $85 \%$, auxiliares de apoyo $88 \%$, otros $84 \%$ ). Se observó solamente un evento adverso serio posiblemente relacionado a la vacuna (cefalea intensa que requirió hospitalización $)^{17}$. La estrategia de vacunación demostró una buena aceptabilidad y un adecuado perfil de seguridad entre los funcionarios de la salud. Como CCI consideramos que la implementación de esta práctica a lo largo de nuestro país sería un avance en el control de la coqueluche. 
Estrategia de vacunación en capullo (“cocooning”)

Con respecto a la estrategia de vacunación de capullo (consistente en la vacunación de las madres en el post-parto inmediato y de todos los contactos cercanos de lactantes bajo 12 meses de edad con dTpa, para reducir el riesgo de transmisión de $B$. pertussis a lactantes), la OMS considera que la evidencia para recomendarla es aún insuficiente ${ }^{1}$. Sin embargo, la ACIP recomienda que los adolescentes y adultos ( v.gr. padres, hermanos, abuelos, cuidadores y funcionarios de la salud) que tienen o tendrán contacto cercano con un lactante bajo 12 meses de edad, debieran recibir una dosis única de dTpa para proteger contra la enfermedad en caso que no hubiesen recibido previamente dTpa. Idealmente, estos adolescentes y adultos debieran recibir dTpa al menos dos semanas antes de comenzar su contacto cercano con el lactante ${ }^{18}$, puesto que la elevación de anticuerpos post vacuna usada como refuerzo requiere al menos 10 días. Lamentablemente, su costo y barreras logísticas para una implementación en la población constituyen aún limitaciones mayores, y nos inclinan a pensar como CCI que no es la mejor estrategia para reducir la coqueluche grave del lactante bajo 3 meses de edad, considerando que por el momento su uso debiera limitarse al control de brotes, y siempre en conjunto con otras estrategias (v.gr. entrega oportuna de quimioprofilaxis a contactos; vacunar a los niños según el Programa Nacional de Inmunizaciones; mantener a los lactantes alejados, tanto como sea posible, de personas con tos o síntomas respiratorios; ventilar ambientes cerrados, entre otras) ${ }^{4}$.

A partir del día 18 de diciembre de 2011 el Ministerio de Salud implementó la estrategia de vacunación en capullo en la Región del Bío-Bío, extendiéndola el 1 de marzo de 2012 a las regiones Metropolitana, Valparaíso y Del Libertador B. O'Higgins. Esta consiste en la vacunación de mujeres puérperas, padres, hermanos y familiares de 12 años y más, incluyendo cuidadoras, que convivan a diario con recién nacidos en las regiones anteriormente mencionadas. La estrategia incluye la vacunación del personal de salud asistencial pediátrico en contacto con recién nacidos y lactantes (Neonatología, Pediatría y UCI neonatal y pediátrica) y se extenderá durante todo 2012. Detalles sobre la implementación de la misma pueden encontrarse en la Circular No8, emitida el 27 de febrero de $2012^{19}$.

\section{Vacunación de la mujer embarazada}

En opinión de la OMS, existe evidencia insuficiente para recomendar la vacunación anti-pertussis en mujeres embarazadas $^{1}$. Sin embargo, la opinión del ACIP es diferente. En este punto, es importante considerar por separado dos aspectos: la seguridad de la vacuna en el embarazo y la posible interferencia inmunológica de los anticuerpos maternos con la respuesta inmune del lactante.

Seguridad de la vacuna. En base a la revisión de datos publicados por el Sistema para Reportar Reacciones
Adversas a las Vacunas (Vaccine Adverse Event Reporting System, VAERS), y otras fuentes no publicadas de laboratorios farmacéuticos Sanofi Pasteur (Adacel $\left.{ }^{\circledR}\right)$ y GlaxoSmithKline (Boostrix $\left.{ }^{\circledR}\right)$, y dos estudios pequeños, ACIP concluyó que los datos disponibles no sugieren una frecuencia elevada o patrones inusuales de eventos adversos en mujeres embarazadas que reciben dTpa, y que los escasos eventos adversos serios reportados eran improbablemente relacionados con la vacuna. Por consiguiente, ACIP recomendó la administración de vacuna dTpa a aquellas mujeres embarazadas que previamente no hayan recibido la vacuna dTpa. La vacuna debe administrarse preferentemente durante el tercer trimestre o tardíamente durante el segundo (después de las 20 semanas de gestación). En caso de no ser administrada durante el embarazo, la vacuna dTpa debe administrarse en el post-parto inmediato ${ }^{18}$.

Interferencia con la respuesta inmune del lactante. Diversos estudios sugieren que en lactantes cuyas madres recibieron vacuna dTpa durante el embarazo, la producción de anticuerpos específicos anti-pertussis por parte del lactante puede verse inhibida por los anticuerpos maternos anti-pertussis. Este fenómeno ocurriría tanto en lactantes que reciben vacunas de células enteras como acelulares. Sin embargo, la importancia clínica de este fenómeno no es del todo clara. La evidencia sugiere que este fenómeno sería breve, debido a que los anticuerpos maternos declinan rápidamente. La vacunación de la embarazada presenta aspectos positivos y negativos. El aspecto positivo sería reducir el riesgo de enfermar y morir en lactantes bajo 3 meses de edad, y a la vez, protección de la propia mujer embarazada; por su parte, el aspecto negativo sería un teórico aumento de incidencia de coqueluche en lactantes de mayor edad. Sin embargo, a esta edad el niño estaría en mejores condiciones para enfrentar la infección por $B$. pertussis ${ }^{18}$.

Por el momento, el CCI considera que la administración de vacuna dTpa a la mujer embarazada constituye una estrategia adecuada y segura para reducir la morbimortalidad por coqueluche en recién nacidos y lactantes, debiendo por ahora limitarse su uso a situaciones de enfrentamiento de brotes, en espera de disponer de mayor evidencia en favor de vacunar a este grupo en forma programática.

\section{En conclusión, el CCI}

- Apoya el desplazamiento a primero básico del segundo refuerzo de vacuna anti-pertussis, difteria y tétanos, junto con el reemplazo de DTwP por dTpa, ya que permitiría extender la duración de la protección.

- Considera importante la incorporación de vacuna dTpa en adolescentes y adultos. 
- Sugiere implementar de manera rutinaria la vacunación anti-pertussis en funcionarios de la salud, especialmente en aquellos que tienen contacto con lactantes con edad inferior a 6 meses y/o pacientes inmunosuprimidos, con el fin de disminuir la incidencia de casos graves de origen nosocomial.

- Considerando que la evidencia a favor de la vacunación de la mujer embarazada y de la estrategia de vacunación en capullo está aún en proceso de generación, sugerimos restringir su implementación por ahora al enfrentamiento de brotes.

\section{Resumen}

En Chile, a comienzos del mes de octubre de 2010 se detectó un aumento en la notificación de casos de coqueluche, dinámica que se ha mantenido a la fecha (abril 2012). El número de casos acumulados durante 2011 ascendió a 2.581 (15,0/100.000 hab.), cifra superior al número de casos registrados durante el período 2008-2010 (2.460 casos). Por su parte, a partir de 2012 la autoridad sanitaria introdujo una modificación en el esquema de vacunación anti-pertussis, consistente en el reemplazo del segundo refuerzo de vacuna antipertussis (DTwP, administrada a los 4 años) y del refuerzo de toxoide diftérico-tetánico (dT, administrado en segundo básico) por la vacuna antipertussis acelular de contenido antigénico reducido (dTpa), a ser administrada en primero básico. El Comité Consultivo de Inmunizaciones considera la modificación adecuada, por cuanto permite extender el tiempo de protección, reduciendo al menos en teoría la infección en escolares mayores y adolescentes -quienes son importantes fuente de contagio de Bordetella pertussis para los lactantes- utilizando una adecuada formulación de vacuna (vacuna antipertussis acelular). Se comenta la evidencia disponible sobre vacunación anti-pertussis en grupos especiales (adolescentes y adultos, funcionarios de la salud y mujeres embarazadas), y la estrategia de vacunación de capullo.

\section{Referencias bibliográficas}

1.- World Health Organization. Pertussis Vaccines: WHO position paper. Weekly Epidemiological Record 2010; 85: 385-400. Disponible en: http://www.who.int/wer/2010/wer8540.pdf [Accedido el 22/04/2012].

2.- Departamento de Epidemiología. Ministerio de Salud, Gobierno de Chile. Informe de Coqueluche Año 2011. Disponible en: http://epi. minsal.cl/epi/html/bolets/reportes/Coqueluche/ Tos_Final_2011.pdf [Accedido el 22/04/2012].

3.- Departamento de Estadísticas e Información en Salud. Ministerio de Salud, Gobierno de Chile. Enfermedades de Notificación Obligatoria (ENO). Disponible en: http://www.deis.cl/ estadisticas-eno/ [Accedido el 22/04/2012].

4.- Departamento de Epidemiología. Ministerio de Salud, Gobierno de Chile. Circular B51/ $\mathrm{N}^{\circ} 27$ Vigilancia Epidemiológica y Medidas de Control de Coqueluche (5 de julio de 2011). Disponible en: http://epi.minsal.cl/epi/html/ normas/circul/Circ.27 Coqueluche2011.pdf [Accedido el 22/04/2012].

5.- Instituto Nacional de Estadísticas. Chile: Proyecciones y estimaciones de población. Total País 1950-2050. Disponible en: http://www. ine.cl/canales/chile_estadistico/demografia_y_ vitales/proyecciones/Informes/Microsoft $\% 20$ Word\%20-\%20InforP_T.pdf [Accedido el 22/04/2012]

6.- Departamento de Epidemiología. Ministerio de Salud, Gobierno de Chile. Informe de Coqueluche. Semana epidemiológica 1 al 15 (al 14/04/2012). Disponible en: http://epi. minsal.cl/epi/html/bolets/reportes/Coqueluche/ Tos_SE152012.pdf [Accedido el 22/04/2012]

7.- Subsecretaría de Salud Pública. División de Prevención y Control de Enfermedades. Departamento de Inmunizaciones. Ordinario B27, №4006. Reemplazo de DTP (vacuna de célula entera) por dpaT (vacuna acelular). Disponible en: http://www.colegiodeenfermeras. cl/Ord.pdf [Accedido el 22/04/2012].

8.- Departamento de Estadísticas e Información en Salud. Ministerio de Salud, Gobierno de Chile. Indicadores Básicos de Salud (2011). Disponible en: http://deis.minsal.cl/deis/indicadores/ INDICADORES_2011.pdf [Accedido el 22/04/2012].

9.- OECD (2011), Health at a Glance 2011: OECD Indicators, OECD Publishing. Disponible en: http://dx.doi.org/10.1787/health_glance-2011-en [Accedido el 22/04/2012].

10.- Wendelboe AM, Van Rie A, Salmaso S, Englund JA. Duration of immunity against pertussis after natural infection or vaccination. Pediatr Infect Dis J. 2005; 24 (5 Suppl): S58-61.

11.- Perret C, Viviani T, Peña A, Abarca K, Ferrés M. Fuente de infección de Bordetella pertussis en lactantes hospitalizados por coqueluche. Rev Méd Chile 2011; 139: 448-54.

12.- Osses R, Díaz O, Saldías F. Infección por Bordetella pertussis: Una causa emergente de tos prolongada en adolescentes y adultos. Rev Chil Enferm Respir 2010; 26: 30-6.

13.- Potin M, Sandoval C, Porte L, Miranda C, Wosniak A, García P. Descripción clínica preliminar de casos de coqueluche confirmados por RPC en la Red de Salud de la Pontificia Universidad Católica de Chile. Rev Chilena Infectol 2011; 28 (Supl 2): S80.

14.- Perret C. Vacuna anti-pertussis para uso en adolescentes y adultos. Rev Chilena Infectol 2006; 23: 257-60.

15.- Muñoz A, Abarca K, Jiménez J, Luchsinger
V, O'Ryan M, Ripoll E, et al. Seguridad de las vacunas que contienen timerosal: Declaración del Comité Consultivo de Inmunizaciones (CCI) de la Sociedad Chilena de Infectología. Rev Chilena Infectol 2007; 24: 372-6.

16.- Centers for Disease Control and Prevention. Updated Recommendations for Use of Tetanus Toxoid, Reduced Diphtheria Toxoid and Acellular Pertussis (Tdap) Vaccine from the Advisory Committee on Immunization Practices, 2010. MMWR Morb Mortal Wkly Rep 2011; 60: 13-5.

17.- Pavéz D, Abarca K, de la Cerda G, Flores C, Cerda J. Reactogenicidad y aceptabilidad de vacuna difteria-tétanos-pertussis acelular (dTpa) en personal de salud en contacto con pacientes pediátricos. Rev Chilena Infectol 2011; 28 (Supl 2): $\mathrm{S} 126$

18.- Centers for Disease Control and Prevention. Updated Recommendations for Use of Tetanus Toxoid, Reduced Diphtheria Toxoid and Acellular Pertussis Vaccine (Tdap) in Pregnant Women and Persons Who Have or Anticipate Having Close Contact with an Infant Aged $<12$ Months --- Advisory Committee on Immunization Practices (ACIP), 2011. MMWR Morb Mortal Wkly Rep 2011; 60: 1424-26.

19.- Subsecretaría de Salud Pública. División de Prevención y Control de Enfermedades. Departamento de Inmunizaciones. Circular N8. Estrategia de vacunación 2012 para control de brote de coqueluche en las regiones Metropolitana, Valparaiso y O'Higgins y extensión de la estrategia en la Región del Bio-Bio (27 de febrero de 2012). Disponible en: http://www.minsal.gob.cl/portal/url/item/ ba4172062b180694e0400101650173d5.pdf [accedido el 22/04/2012]. 